INPLASY

PROTOCOL

To cite: Li et al. Music intervention on physiological and psychological responses of patients with cancer: a meta- analysis protocol. Inplasy protocol 202110082. doi:

10.37766/inplasy2021.1.0082

Received: 21 January 2021

Published: 21 January 2021

Corresponding author:

Aimin Zang

zou5867@163.com

Author Affiliation:

Affiliated Hospital of Hebei

University

Support: 2017Q002.

Review Stage at time of this submission: The review has not yet started.

\section{Music intervention on physiological and psychological responses of patients with cancer: a meta- analysis protocol}

Li, N1; Liu, D2; Zhang, L3; Zhao, L4; Guo, F5; Zang, A6.

Review question / Objective: Cancer is a life-threatening condition and also one of the biggest challenges facing human health and the medical community. This meta-analysis was to investigate the effects of music intervention on physiological and psychological responses of patients with cancer.

Condition being studied: Patients with cancer.

INPLASY registration number: This protocol was registered with the International Platform of Registered Systematic Review and Meta-Analysis Protocols (INPLASY) on 21 January 2021 and was last updated on 21 January 2021 (registration number INPLASY202110082).

Conflicts of interest:

None.

\section{INTRODUCTION}

Review question / Objective: Cancer is a life-threatening condition and also one of the biggest challenges facing human health and the medical community. This metaanalysis was to investigate the effects of music intervention on physiological and psychological responses of patients with cancer.
Condition being studied: Patients with cancer.

\section{METHODS}

Participant or population: cancer patients aged 18 to 75 years.

Intervention: Music intervention.

Comparator: placebo. 
Study designs to be included: RCTs.

Eligibility criteria: (1) Patients: cancer patients aged 18 to 75 years; (2) Intervention: music intervention; (3) Control: placebo; (4) Outcomes: state-trait anxiety inventory, self-rating anxiety scale, self-rating depression scale and visual analogue scale; and (5) Study design: randomized controlled trials.

Information sources: Electronic databases.

Main outcome(s): State-trait anxiety inventory, self-rating anxiety scale, selfrating depression scale and visual analogue scale.

Quality assessment / Risk of bias analysis: Two examiners (Nan Li and Dan Liu) independently in blind performed quality and risk of bias assessment. The third expert would join in to discuss and resolve divergence. Risk of bias assessment was assessed using the Cochrane Collaboration's tool for assessing risk of bias in randomized trials. Because the number of studies included in this metaanalysis of randomized, double or tripleblind, controlled clinical trials was eight less than ten, the funnel plot wasn't used to explore publication bias.

Strategy of data synthesis: Review Manager software (version 5.3; Nordic Cochrane Centre, The Cochrane Collaboration,) was used to perform data synthesis and analysis. For dichotomous outcomes, that is adverse events, RRs with corresponding $95 \%$ confidence intervals (Cls) was used to estimate pool effects. For continuous outcomes, when included studies not directly reported data used to aggregate effects12. These data were calculated from $95 \% \mathrm{Cls}$ and changes from baseline to 6,12 months. Further, when there were more than two intervention groups or control groups in an included trial, we joined two groups into a single group. We used mean differences (MDs) and $95 \% \mathrm{Cls}$ for outcomes with the same measures; standard mean differences (SMDs) and 95\% Cls for outcomes with different measures. A SMDs of 0.20 is considered a small difference between the experimental and the control group; 0.50, a moderate difference; and 0.80 , a large difference. Statistical heterogeneity among included studies was assessed with 12 statistic: $0 \%$ to $40 \%$, might not be important; $30 \%$ to $60 \%$, may represent moderate heterogeneity; $50 \%$ to $90 \%$, represent substantial heterogeneity; $75 \%$ to $100 \%$ : considerable heterogeneity. As heterogeneity in risk ratios (RRs), MDs, SMDs is $0 \%$ or might not be important, fixed-effect 176 was used to pool the results. All statistical tests undertaken were 2-sided and considered a $P$ value $\leq 0.05$ or a $95 \%$ Cls that excluded a null result as statistically significant.

Subgroup analysis: None.

Sensibility analysis: None.

Country(ies) involved: China.

Keywords: Music intervention; Cancer; Outcomes; Protocol.

Contributions of each author:

Author 1 - Nan Li.

Author 2 - Dan Liu.

Author 3 - Lei Zhang.

Author 4 - Linshan Zhao.

Author 5 - Fan Guo.

Author 6 - Aimin Zang. 